\title{
Review: "distant healing" is often effective for improving patient outcomes
}

Astin JA, Harkness E, Ernst E. The efficacy of "distant healing": a systematic review of randomized trials. Ann Intern Med 2000 Jun 6;132:903-10.

QUESTION: Is "distant healing," defined as prayer, mental healing, therapeutic touch, or spiritual healing, effective treatment for any medical condition?

\section{Data sources}

Studies were identified by searching Medline, PsycLIT, EMBASE/Excerpta Medica, CISCOM, and the Cochrane Library from their inception to 1999 with terms related to spiritual healing, mental healing, faith healing, prayer, therapeutic touch, Reiki, distant healing, psychic healing, and external qigong and with the terms clinical trials, controlled clinical trials, and randomised controlled trials. Bibliographies of relevant studies were scanned, leading researchers in the field were contacted, and personal files were searched.

\section{Study selection}

Randomised controlled trials were selected if distant healing was compared with placebo, sham, or otherwise "patient blindable" or adequate control interventions; clinical rather than experimental investigations were done; humans with any medical conditions were studied; and publication was in peer reviewed journals.

\section{Data extraction}

Data were extracted on year of publication, design, study quality, sample size, experimental and control interventions, and results.

\section{Main results}

23 studies of 2774 patients met inclusion criteria. Methodological quality was fairly high (mean score 3.6, maximum possible 5). Effect sizes were calculated and averaged, but a formal meta-analysis was not done because of heterogeneity. Prayer was evaluated in 5 studies: 2 showed a positive treatment effect in $\geqslant 1$ outcome, and 3 showed no effect. The mean effect size for 4 studies was $0.25(\mathrm{p}=0.009)$. 11 trials evaluated non-contact therapeutic touch: 7 showed a positive treatment effect in $\geqslant 1$ outcome, 3 showed no effect, and 1 had a negative effect. The mean effect size for 10 studies was $0.63 \quad(p=0.003)$. Other studies evaluated "distance or distant healing" (4 studies), paranormal healing (1 study), psychokinetic influence (1 study), and remote mental healing ( 1 study). 4 of these trials showed a positive treatment effect, and 3 showed no effect. The mean effect size for 5 studies was $0.38(\mathrm{p}=0.07)$.

For all 23 studies, $13(57 \%)$ had a positive treatment effect, 9 had no effect, and 1 had a negative effect. The mean effect size across 16 of the 23 trials was 0.4 $(\mathrm{p}<0.001)$. Homogeneity was shown for the studies of prayer and other forms of distant healing but not for studies of therapeutic touch. Calculations showed that 63 additional studies with no effect would have to be incorporated into the analyses before the overall results became statistically non-significant.

\section{Conclusion}

Distant healing, including prayer and therapeutic touch, is often effective in improving patient outcomes.

\section{COMMENTARY}

Most Americans believe that such distant healing interventions as prayer or therapeutic touch are effective. ${ }^{1}$ Astin $e t$ al carefully and systematically reviewed research on distant healing. Despite a comprehensive search, they identified only 23 investigations that met their criteria. This is not surprising. 1 recent study found that only $0.08 \%$ of all Medline citations involved religious or spiritual issues. ${ }^{2}$ Why has such a widely used intervention received so little scrutiny? It is difficult to standardise a distant healing intervention. We cannot readily measure the skill of a distant healer or quantify the "dose" of an intervention, nor do we even know if such variables are relevant. In addition, many scientists find it completely implausible that any effect exists for distant healing and therefore are unlikely to initiate research in such a controversial area. We could, however, resolve many of the unanswered questions about distant healing. Even if disagreement exists about its mechanism, research can still describe the intervention and measure its effect.

The evidence, although scanty, suggests but does not prove that distant healing actually heals. The incorporation of any form of distant healing into clinical practice would require a major shift in our beliefs about both healing and science. Do we have enough faith in the scientific method to prescribe interventions with proven efficacy if we do not understand their mechanism? We are in a position similar to that of Alexander Fleming when he saw the effect of a penicillin mold on bacteria in a petri dish. One of Fleming's colleagues said that it "seems like a miracle," and another replied, "Of course, it does. It is a miracle." ${ }^{3}$ The miracle of one generation is the technology of the next.

The only thing that is certain about the effect of distant healing is that further research is justified. Medical science will not advance if we only explore what we already know. Michael J Baime, MD University of Pennsylvania School of Medicine Philadelphia, Pennsylvania, USA

1 Wallis C. Faith and healing: can prayer, faith and spirituality really improve your physical health? A growing and surprising body of scientific evidence says they can. Time 1996; 147:58.

2 Lukoff D, Provenzano R, Lu F, et al. Religious and spiritual case reports on MEDLINE: a systematic analysis of records from 1980 to 1996. Altern Ther Health Med 1999;5:64-70.

3 Brownlee S. Alexander Fleming: medicine's accidental hero. U.S. News \& World Report. Aug. 17, 1998. http:// www.usnews.com/usnews/issue//17flem.htm.
Sources of funding: National Institutes of Health; Wellcome Trust; Maurice Laing Foundation.

For correspondence: DrJA Astin, Complementary Medicine Program, Kernan Hospital Mansion, 2200

Kernan Drive, Baltimore, $M D$ 21207-6697, USA. Fax +4104486875 . 